\title{
Using Small RNA Deep Sequencing Data to Detect Human Viruses
}

\author{
Fang Wang, ${ }^{1}$ Yu Sun, ${ }^{2}$ Jishou Ruan, ${ }^{3}$ Rui Chen, ${ }^{4}$ Xin Chen, ${ }^{2}$ Chengjie Chen, ${ }^{5}$ Jan F. Kreuze, \\ ZhangJun Fei, ${ }^{7}$ Xiao Zhu, ${ }^{8}$ and Shan Gao ${ }^{2}$
}

${ }^{1}$ Department of Gynaecology, The Second Hospital, Tianjin Medical University, Tianjin 300211, China

${ }^{2}$ College of Life Sciences, Nankai University, Tianjin 300071, China

${ }^{3}$ School of Mathematical Sciences, Nankai University, Tianjin 300071, China

${ }^{4}$ Tianjin Institute of Agricultural Quality Standard and Testing Technology, Tianjin Academy of Agricultural Sciences, Tianjin 300381, China

${ }^{5}$ College of Horticulture, South China Agricultural University, Guangzhou, Guangdong 510642, China

${ }^{6}$ International Potato Center (CIP), Apartado 1558, Lima 12, Peru

${ }^{7}$ Boyce Thompson Institute for Plant Research, Cornell University, Ithaca, NY 14853, USA

${ }^{8}$ Guangdong Provincial Key Laboratory of Medical Molecular Diagnostics, Dongguan Scientific Research Center, Guangdong Medical University, Dongguan, Guangdong 523808, China

Correspondence should be addressed to Xiao Zhu; bioxzhu@yahoo.com and Shan Gao; gao_shan@mail.nankai.edu.cn

Received 7 November 2015; Revised 13 January 2016; Accepted 3 February 2016

Academic Editor: Jozef Anné

Copyright (c) 2016 Fang Wang et al. This is an open access article distributed under the Creative Commons Attribution License, which permits unrestricted use, distribution, and reproduction in any medium, provided the original work is properly cited.

\begin{abstract}
Small RNA sequencing (sRNA-seq) can be used to detect viruses in infected hosts without the necessity to have any prior knowledge or specialized sample preparation. The sRNA-seq method was initially used for viral detection and identification in plants and then in invertebrates and fungi. However, it is still controversial to use sRNA-seq in the detection of mammalian or human viruses. In this study, we used 931 sRNA-seq runs of data from the NCBI SRA database to detect and identify viruses in human cells or tissues, particularly from some clinical samples. Six viruses including HPV-18, HBV, HCV, HIV-1, SMRV, and EBV were detected from 36 runs of data. Four viruses were consistent with the annotations from the previous studies. HIV-1 was found in clinical samples without the HIV-positive reports, and SMRV was found in Diffuse Large B-Cell Lymphoma cells for the first time. In conclusion, these results suggest the sRNA-seq can be used to detect viruses in mammals and humans.
\end{abstract}

\section{Introduction}

Infection by pathogens is one of the main risk factors for many diseases [1-4], particularly for cancers. In 2008, approximately two million new cancer cases $(16 \%)$ worldwide were caused by pathogen infection. Most cancers inducing infectious agents were viruses [5], including Epstein-Barr virus (EBV), hepatitis B and C virus (HBV and HCV, resp.), Kaposi sarcoma herpes virus (KSHV, also known as human herpes virus type $8, \mathrm{HHV}-8$ ), human immunodeficiency virus type 1 (HIV-1), human papillomavirus type 16 (HPV-16), and human T-cell lymphotropic virus type 1 (HTLV-1). Therefore, the rapid and accurate detection and identification of these viruses is essential to human health. Conventional detection methods (e.g., ELISA, PCR, or microarrays) cannot be used in some cases due to failure to satisfy certain requirements (e.g., prior knowledge of the potential pathogen or the ability to cultivate and purify the pathogen [6]). In addition, they are time-consuming and difficult to use in detection of highly divergent or novel viruses.

To overcome these limitations, next generation sequencing (NGS) technologies have been applied for virus and viroid discovery in plants and animals [7,8]. Compared to other NGS based methods requiring the use of viral enrichment and concentration procedures [7], the small RNA sequencing (sRNA-seq) based method simplifies the virus detection, with the aid of virus fragments enriched by the RNA interference (RNAi) mechanism. RNAi is a cytoplasmic 
cell surveillance system which recognizes double-stranded RNA (dsRNA) and specifically destroys single-stranded RNA and dsRNA molecules homologous to the dsRNA inducer, using small interfering RNAs (siRNAs) as a guide [9]. The abundant siRNAs accumulated during the RNAi process facilitate virus detection and the study of RNAi mechanism. RNAi has been proposed as a key antiviral intrinsic immune response in plants, nematodes, and arthropods [10]. Based on such theory, the sRNA-seq method was originally used for viral detection and identification in plants $[8,11,12]$ and in invertebrates [13-15], but not in mammals or humans. There was evidence that antiviral RNAi functions in mammalian germ cells and embryonic stem cells (ESCs), as well as some carcinoma cell lines [10]. No evidence had been provided to prove RNAi functions in mammalian somatic cells until Li et al's work was published [16]. Although Li et al. discovered low level siRNA duplexes in the baby hamster kidney 21 cells, the role of RNAi in viral defence in mammalians remains controversial. Therefore, using sRNA-seq to detect viruses in mammals and humans is a highly promising but hard topic.

In this study, we used 931 sRNA-seq runs of data from the NCBI SRA database [17] to detect and identify viruses in human cells or tissues, particularly from some clinical samples. These tissues came from saliva, tongue, laryngopharynx, oropharynx, prefrontal cortex, liver, cervix, serum, plasma, lymph, and so forth. As a result, six viruses including HPV-18, HBV, HCV, HIV-1, SMRV (squirrel monkey retrovirus), and EBV were detected from 36 runs of data. In brief, the existence of HPV-18, HBV, HCV, and EBV was consistent with the findings from the original studies, whereas HIV-1 and SMRV had not been identified previously in the experimental samples. The nucleotide polymorphism, read-enriched regions (hotspots), and RNAi responses of detected viruses were analyzed, following the detection of these viruses.

\section{Materials and Methods}

Using NCBI SRA advanced searching tools (http://www.ncbi .nlm.nih.gov/sra/advanced), we retrieved 2,820 runs of data by the combined keywords including Illumina, small RNA, and Homo sapiens (November 1, 2014). We subsequently filtered these data based on the following criteria: (1) to remove non-small RNA-seq data by reading the annotations; (2) to remove data containing keyword "cell line"; (3) to remove data from cDNA library selection during library construction. Ultimately, we used 931 runs of data from 42 previous studies in this study (Table 1).

The software Fastq_clean [18] was used for sRNA data cleaning and quality control. To detect and identify viruses using sRNA-seq data, we developed an automatic pipeline using Perl scripts. This pipeline had performed well in the detection and identification of plant and insect viruses in our previous studies $[12,19-21]$. The pipeline integrated three sequence databases: The first one was an rRNA database, which was built based on the SILVA ribosomal RNA gene database [22]. The second one was the human host genome for the subtraction of host genome sequences. The last one contained the Vertebrata viral sequences constructed from the NCBI GenBank database, version 197. The relationship information between the virus genus and the host was from the International Committee on Taxonomy of Viruses (ICTV). For some virus genera which did not have host information assigned to them, we were able to assign host categories after reading their NCBI annotations.

For each detected virus, we assigned a putative reference genome from the NCBI GenBank database to represent the virus (Supplementary File 1 in Supplementary Material available online at http://dx.doi.org/10.1155/2016/2596782). We used the reference genome coverage and the average depth to quantify the detected viruses. The genome coverage represents the proportion of read-covered positions against the genome length. The average depth is equal to the total base pairs of the aligned reads divided by the read-covered positions on the reference genome (Tables 2 and 3 ).

\section{Results and Discussion}

3.1. HPV-18 from HeLa Cell Lines. To test our virus detection pipeline, we used HeLa cell line data from the previous study SRP001381 as positive controls (Table 1). The HeLa cell line, derived from cervical cancer cells of the patient Henrietta Lacks, contains HPV-18, one of the carcinogenic HPV genotypes. In this study, HPV-18 was detected in all of the three runs of data (SRR031635, SRR031636, and SRR031637). The assembled HPV-18 in the data SRR031636 covered $74.1 \%$ of the reference genome M20325 with an average depth 8.5 . The 19 long viral contigs ( $\geq 40 \mathrm{bp}$ ) covered $62.5 \%$ of the reference genome with a uniform distribution (Supplementary File 1).

3.2. HBV and HCV from Human Liver and HCC Tissues. Chronic hepatitis $B$ virus (HBV) is one of the first viruses to be causally linked to a human tumor and is a major global cause of hepatocellular carcinoma (HCC). HBV, hepatitis C virus (HCV), and cirrhosis between them contribute to the genesis of almost all global HCCs [23]. Conventional clinical tests use markers at the protein level, including the $\mathrm{HBV}$ surface antigen ( $\mathrm{HBsAg}$ ), $\mathrm{HBV}$ envelope antigen ( $\mathrm{HBeAg}$ ), and $\mathrm{HBV}$ core antigen ( $\mathrm{HBcAg}$ ) and their antibodies from the patients' serum. However, these protein markers are not always present for various reasons [23].

In the previous study SRP002272 from the NCBI SRA database (Table 2), 15 clinical samples had been sequenced including three normal liver tissues, one $\mathrm{HBV}$-infected liver tissue, one severe chronic hepatitis B liver tissue, two HBVpositive HCC tissues, one HCV-positive HCC tissue, and one HCC tissue without HBV or HCV [24]. In this study, the detection and identification results in 15 runs of data were consistent with the findings from the previous study SRP002272 with one exception SRR039619 (Table 2). The sRNA data SRR039619 from a HBV-positive HCC patient should have contained HBV but it was not found by our pipeline. SRR039619 contained 9,161,157 reads, which possibly were not deep enough to catch adequate virus derived small RNAs (vsRNAs) for detection.

The assembled HBV in the data SRR039620 covered $54.6 \%$ of the reference genome JQ688404 with an average depth 6 (Supplementary File 1). In the data SRR039620, seven 
TABLE 1: The 42 previous studies from the SRA database.

\begin{tabular}{|c|c|c|c|}
\hline Study ID & Runs & Sample source & Disease \\
\hline DRP000998 & 3 & Whole saliva, salivary exosome & Healthy \\
\hline ERP001908 & 63 & Tongue, laryngopharynx, oropharynx & HNSCC \\
\hline ERP004592 & 23 & Prefrontal cortex & Huntington's disease \\
\hline SRP001381 & 3 & HeLa cell line & HPV18(+) \\
\hline SRP002118 & 14 & Hek293T cell line & NA \\
\hline SRP002272 & 15 & Liver & $\mathrm{HBV}(+), \mathrm{HCV}(+), \mathrm{HCC}$ \\
\hline SRP002326 & 38 & Cervical tumor & Cervical cancer \\
\hline SRP002402 & 3 & Sperm & Healthy \\
\hline SRP007825 & 67 & Skin & Psoriasis \\
\hline SRP008258 & 2 & Hek293, HeLa cell line & NA \\
\hline SRP009246 & 4 & Primary human fibroblast & NA \\
\hline SRP014020 & 20 & Thyroid tumor & Follicular thyroid adenoma \\
\hline SRP017809 & 4 & Dorsolateral prefrontal cortex & Healthy \\
\hline SRP017979 & 4 & Colorectal tumor & Colorectal cancer \\
\hline SRP018255 & 35 & Plasma, serum, placenta & Healthy \\
\hline SRP021130 & 20 & Cerebral cortex & $\begin{array}{l}\text { FTLD, PSP, BHS, DLB, } \\
\text { Alzheimer's disease }\end{array}$ \\
\hline SRP021193 & 40 & Heart & NIC, IC \\
\hline SRP021911 & 12 & Cumulus granulosa cell, mural granulosa cell & NA \\
\hline SRP021924 & 5 & Brain frontal cortex & NA \\
\hline SRP022043 & 70 & Blood & Alzheimer's disease \\
\hline SRP022054 & 26 & $\begin{array}{l}\text { Sigma, liver, coecum, colon ascendens, lymph } \\
\text { node }\end{array}$ & Colorectal cancer \\
\hline SRP026081 & 2 & Penicillium marneffei & NA \\
\hline SRP026558 & 2 & PBMC & Osteopetrosis \\
\hline SRP026562 & 11 & Prefrontal cortex & Alzheimer's disease \\
\hline SRP027589 & 42 & Serum & Breast cancer \\
\hline SRP028291 & 78 & ACA, ACC tumor, adrenal tissue & ACA, ACC \\
\hline SRP028738 & 16 & MiRQC, serum, liver & NA \\
\hline SRP029599 & 9 & FFPE, serum & Nonkeratinizing NPC, NPC \\
\hline SRP032650 & 4 & Serum & Latent PTB, PTB \\
\hline SRP032953 & 12 & Alpha cell, beta cell, whole islet & Type 2 diabetes mellitus \\
\hline SRP033505 & 3 & Plasma & Healthy \\
\hline SRP033566 & 185 & $\begin{array}{l}\text { Connective tissue, plasma, neuronal tissue, } \\
\text { primary cell, cardiac muscle, epithelium, skeletal } \\
\text { muscle }\end{array}$ & DCM, IC \\
\hline SRP034547 & 4 & Primary fibroblast & Microcephaly \\
\hline SRP034586 & 24 & Serum, PBMC & Healthy \\
\hline SRP034590 & 14 & Plasma & NA \\
\hline SRP034654 & 12 & $\begin{array}{l}\text { Tensor fascia lata, quadricep vastus, vastus } \\
\text { externe, rhomboid, iliopsoas }\end{array}$ & FSHD \\
\hline SRP034698 & 8 & Skin, lymph node & MCC, SCC, melanoma, BCC \\
\hline SRP040421 & 12 & Exosome in human semen & Healthy \\
\hline SRP041082 & 2 & Seminal fluid & Prostate cancer \\
\hline SRP046046 & 12 & Lymphoblastoid & $\begin{array}{l}\text { DLBCL, Burkitt's lymphoma, } \\
\text { EBV }(+)\end{array}$ \\
\hline SRP046234 & 2 & Breast epithelium & Triple negative breast cancer \\
\hline
\end{tabular}


TABLE 1: Continued.

\begin{tabular}{|c|c|c|c|}
\hline Study ID & Runs & Sample source & Disease \\
\hline SRP048290 & 6 & Platelet & $\mathrm{He}$ \\
\hline \multicolumn{4}{|c|}{$\begin{array}{l}\text { "Study ID" is uniq for each high-throughput project in the NCBI SRA database. ACA: adrenal cortical adenoma, ACC: adrenal cortical carcinoma, BCC: } \\
\text { Basal Cell Carcinoma, BHS: bilateral hippocampal sclerosis, DCM: Dilated Cardiomyopathy, DLB: dementia with Lewy bodies, DLBCL: Diffuse Large B- } \\
\text { Cell Lymphoma, FSHD: Facioscapulohumeral Muscular Dystrophy, FTLD: frontotemporal lobar dementia, HCC: HBV-related hepatocellular carcinoma, } \\
\text { HNSCC: Head and Neck Squamous Cell Carcinoma, IC: Ischemic Cardiomyopathy, MCC: Merkel Cell Carcinoma, NIC: Nonischemic Cardiomyopathy, } \\
\text { NPC: nasopharyngeal carcinoma, PBMC: Peripheral Blood Mononuclear Cell, PSP: Progressive Supranuclear Palsy, PTB: Pulmonary Tuberculosis, and SCC: } \\
\text { Squamous Cell Carcinoma. }\end{array}$} \\
\hline
\end{tabular}

TABLE 2: HBV and HCV from the SRP002272 study.

\begin{tabular}{|c|c|c|c|c|}
\hline Run ID & Sample_Source & Reference & $\operatorname{Cov}(\%)$ & Depth \\
\hline SRR039611 & Human Normal Liver Tissue & NA & NA & NA \\
\hline SRR039612 & Human Normal Liver Tissue & NA & NA & NA \\
\hline SRR039613 & Human Normal Liver Tissue & NA & NA & NA \\
\hline SRR039614 & HBV-Infected Liver Tissue & JQ688405 & $423(13.2)$ & 3.0 \\
\hline SRR039615 & Severe Chronic Hepatitis B Liver Tissue & NA & NA & NA \\
\hline SRR039616 & HBV(+) Distal Tissue & NA & NA & NA \\
\hline SRR039617 & HBV(+) Adjacent Tissue & NA & NA & NA \\
\hline SRR039618 & $\mathrm{HBV}(+)$ Side Tissue & NA & NA & NA \\
\hline SRR039619* & HBV(+) HCC Tissue & NA & NA & NA \\
\hline SRR039620 & HBV(+) Adjacent Tissue & JQ688404 & $1756(54.6)$ & 6.0 \\
\hline SRR039621 & HBV(+) HCC Tissue & GQ475344 & $321(10)$ & 1.5 \\
\hline SRR039622 & HCV(+) Adjacent Tissue & D85516 & $1032(10.8)$ & 1.8 \\
\hline SRR039623 & $\mathrm{HCV}(+) \mathrm{HCC}$ Tissue & GU133617 & $805(8.3)$ & 8.0 \\
\hline SRR039624 & HBV(-) HCV(-) Adjacent Tissue & NA & NA & NA \\
\hline SRR039625 & HBV(-) HCV(-) HCC Tissue & NA & NA & NA \\
\hline
\end{tabular}

"Run ID" is uniq for each high-throughput fastq file in the NCBI SRA database. "Reference" uses the NCBI GenBank accession number. "Cov (\%)" and "Depth" represent the genome coverage and the average depth, respectively. "Side Tissue" is close to the border between the tumor tissues and the normal tissues but $0-2 \mathrm{~cm}$ far from the tumor tissues. "Adjacent Tissue" is the normal tissues $2-5 \mathrm{~cm}$ far from the tumor tissues. "Distal Tissue" is the normal tissues at least $10 \mathrm{~cm}$ far from the tumor tissues. "SRR039619*" should have contained HBV but it was not found by our pipeline.

TABLE 3: SMRV and EBV from the SRP046046 study.

\begin{tabular}{|c|c|c|c|c|}
\hline Run ID & Sample_Source & Reference & Cov (\%) & Depth \\
\hline SRR1563015 & DLBCL & M23385 & $8714(99.2)$ & 146.1 \\
\hline SRR1563017 & DLBCL Exosome & M23385 & $8732(99.4)$ & 494.5 \\
\hline SRR1563018 & $\mathrm{EBV}(+) \mathrm{BL}$ & KC207813 & $2765(1.6)$ & 29.2 \\
\hline SRR1563056 & EBV $(+)$ BL Exosome & KC207813 & $33107(19.3)$ & 9.6 \\
\hline SRR1563057 & $\mathrm{EBV}(-) \mathrm{BL}$ & NA & NA & NA \\
\hline SRR1563058 & EBV (-) BL Exosome & NA & NA & NA \\
\hline SRR1563059 & $\mathrm{EBV}(+) \mathrm{LCL}$ & KC207813 & $13757(8)$ & 358.2 \\
\hline SRR1563060 & EBV(+) LCL Exosome & M80517 & $7444(4)$ & 288.8 \\
\hline SRR1563061 & $\mathrm{EBV}(+) \mathrm{LCL}$ & M80517 & $18688(10.2)$ & 151.1 \\
\hline SRR1563062 & EBV $(+)$ LCL Exosome & KC207814 & $7931(4.6)$ & 198.2 \\
\hline SRR1563063 & $\mathrm{EBV}(+) \mathrm{LCL}$ & M80517 & $37898(20.6)$ & 52.8 \\
\hline SRR1563064 & EBV (+) LCL Exosome & M80517 & $57850(31.4)$ & 17.6 \\
\hline
\end{tabular}

"Run ID" is uniq for each high-throughput fastq file in the NCBI SRA database. "Reference" uses the NCBI GenBank accession number. "Cov (\%)" and "Depth" represent the genome coverage and the average depth, respectively.

long viral contigs $(\geq 40 \mathrm{bp})$ covered the $\mathrm{HBV} \times(\mathrm{HBx}), \mathrm{HBV}$ core $(\mathrm{HBc})$, and $\mathrm{HBV}$ polymerase $(\mathrm{HBp})$ gene regions but did not cover the HBV surface (HBs) gene region. The long viral contigs ( $\geq 40 \mathrm{bp}$ ) in the data SRR039614 and SRR039621 only covered the HBx gene region. The assembled HCV in the data SRR039622 covered $10.8 \%$ of the reference genome D85516 with an average depth 1 (Supplementary File 1). HCV was also detected in the data SRR039623 with genome coverage 8.3\% and average depth 1 .

3.3. HIV-1 from Breast Cancer Patients. HIV as a member of the genus Lentivirus causes acquired immunodeficiency syndrome (AIDS). As technology evolves, HIV testing assays are being improved on sensitivity and specificity [25]. However, 
the tests still provide false negative results due to the diagnostic window or other reasons [25]. In the previous study SRP027589 from the NCBI SRA database (Table 1), 42 samples had been sequenced for the discovery and profiling of circulating microRNAs in the serum of 42 stage II-III locally advanced and inflammatory breast cancer (BC) patients [26]. These patients received neoadjuvant chemotherapy (NCT) followed by surgical tumor resection. However, no AIDS or HIV-positive results of these patients had been reported in the previous study SRP027589. In this study, HIV-1 was detected at a very high level in the data SRR941591. The assembled HIV-1 in the data SRR941591 covered 39.3\% of the reference genome M19921 with an average depth 210.1 (Supplementary File 1). As far as we know, this was the first time to report the detection of HIV-1 using sRNA data from clinical samples.

3.4. SMRV and EBV from B Cells and Exosomes. SMRV, an endogenous virus of squirrel monkeys, had been isolated by cocultivation of squirrel monkey lung cells with canine cells [27]. In previous studies, SMRV had been detected in Burkitt's lymphoma (BL) cell lines [28]. Specifically, the insertion of the incomplete SMRV proviral genomes had been detected in Namalwa cell lines [29]. However, we found no reports that SMRV had been detected in the Diffuse Large B-Cell Lymphoma (DLBCL). To the best of our knowledge, DLBCL had only been reported to be caused by EBV [30], HCV [31], HIV [32], and SV40 (Simian Virus 40) [33].

In this study, SMRV was detected in the data SRR1563015 and SRR1563017 (Table 3). The assembled SMRV in these two runs of data covered $99.2 \%$ and $99.4 \%$ of the reference genome M23385 at an average depth of 146.1 and 494.5, respectively. In the data SRR1563017, the longest viral contig was assembled to have a length of $6,760 \mathrm{bp}$ and an identity $99 \%(6,751 / 6,764)$ of the reference sequence M23385 (Supplementary File 1). As far as we know, this was the first time to report the detection of SMRV using sRNA data from DLBCL samples.

Epstein-Barr virus (EBV) has been firmly linked to some cancers and proliferative diseases, including Burkitt's lymphoma (BL), nasopharyngeal carcinoma, immunoblastic lymphoma, a subset of gastric carcinomas, rare T- and NKcell lymphomas or leiomyosarcoma, acute infectious mononucleosis, and Hodgkin's disease. Almost 100\% of BL cases in Equatorial Africa carry EBV. Children infected early in life with the highest antibody titres to the virus are at the highest risk of developing the tumor [34]. EBV-positive BL predominant in Africa and EBV-negative BL predominant in Europe and/or the United States have different causation and characteristics [34].

In the previous study SRP046046 from the NCBI SRA database, 12 samples had been sequenced to distinguish the small RNA composition in six B cells from their exosomes. Six B cells included three EBV-positive lymphoblastoid B cells (LCLs), one EBV-positive Burkitt's lymphoma (BL) cell, one EBV-negative BL cell, and one Diffuse Large B-Cell Lymphoma (DLBCL) cell. As a result, EBV had been detected from two EBV-positive BL samples and six EBV-positive LCL samples (Table 3). In this study, EBV was detected in the data SRR1563018, SRR1563056, SRR1563059, SRR1563060, SRR1563061, SRR1563062, SRR1563063, and SRR1563064. This finding confirmed the results in the previous study SRP046046. However, the reference genome coverage by vsRNAs was uneven in eight runs of data varying from $1.6 \%$ to $31.4 \%$. This large variance could result from sample extraction, small RNA library construction, sequencing quality, or sequencing depth. In the data SRR1563063, the assembled EBV contigs covered $20.6 \%$ of the reference genome M80517 (Supplementary File 1).

3.5. Nucleotide Polymorphism, Hotspots, and RNAi Responses. The plant sRNA-seq data had been shown to contain adequate information for studying nucleotide polymorphism of the actual virus [35]. Among the six human viruses found in this study, HIV-1 in the data SRR941591 showed the highest nucleotide polymorphism rate covering $2.66 \%(155 / 5,831)$ of the genomic positions (Figure 1), as compared to SMRV in the data SRR1563017, EBV in the data SRR1563063, and HPV18 in the data SRR031636 covering only $0.41 \%(36 / 8,732)$, $0.29 \%(110 / 37,898)$, and $0.13 \%(3 / 2,324)$ of the genomic positions, respectively (Supplementary File 2). HIV-1 is a single-stranded RNA (ssRNA) reverse-transcribing virus. HIV reverse transcriptase has been shown to be exceptionally inaccurate [36] and may explain the high polymorphism rates observed in this study. HPV-18 and EBV are double-stranded DNA (dsDNA) viruses which have low error rates during their replication. SMRV, as ssRNA retrovirus, was expected to have a high nucleotide polymorphism rate but this was not reflected in these data. HBV and HCV showed no polymorphism whatsoever, probably due to the low sequencing depth.

Consistent with our previous results in plant virus detection, the distribution of vsRNA coverage over the human virus genomes was not even, with some read-enriched regions (hotspots) in the vsRNA-covered regions on both of the positive and negative strands (Supplementary File 3). In HPV-18, HBV, HCV, and SMRV, the vsRNA-covered region on the positive strand was more than nine times larger than the vsRNA-covered region on the negative strand, while HIV-1 and EBV had little difference between vsRNA-covered regions over the positive and negative strands. Using the data SRR941591 as an example (Figure 1), the number of bases covered by vsRNA reads on the HIV-1 positive strand against the negative strand was 4,961 bp to 3,945 bp with overlap $52.74 \%(3,075 / 5,831)$. There were three obvious hotspots on the putative HIV-1 reference M19921. The first (779-810 bp) and second (2,017-2,045 bp) hotspot resided on the HIV-1 positive strand. Different from the first and second hotspot, the third hotspot $(12,006-12,044 \mathrm{bp})$ consisted of positiveand negative-strand vsRNAs.

To investigate the RNAi responses using 36 viruscontaining runs of data, we analyzed the length distribution of the reads aligned to the virus reference sequences. Viral small RNA read lengths of HIV-1 in the data SRR941591 had the distribution pattern expected from a RNAi response, similar to what had been found in previous studies [16]. This pattern consists of positive- and negative-strand vsRNAs with countable values at the $21,22,23$, and $24 \mathrm{bp}$ read length (Figure 2). Another characteristic of RNAi responses 


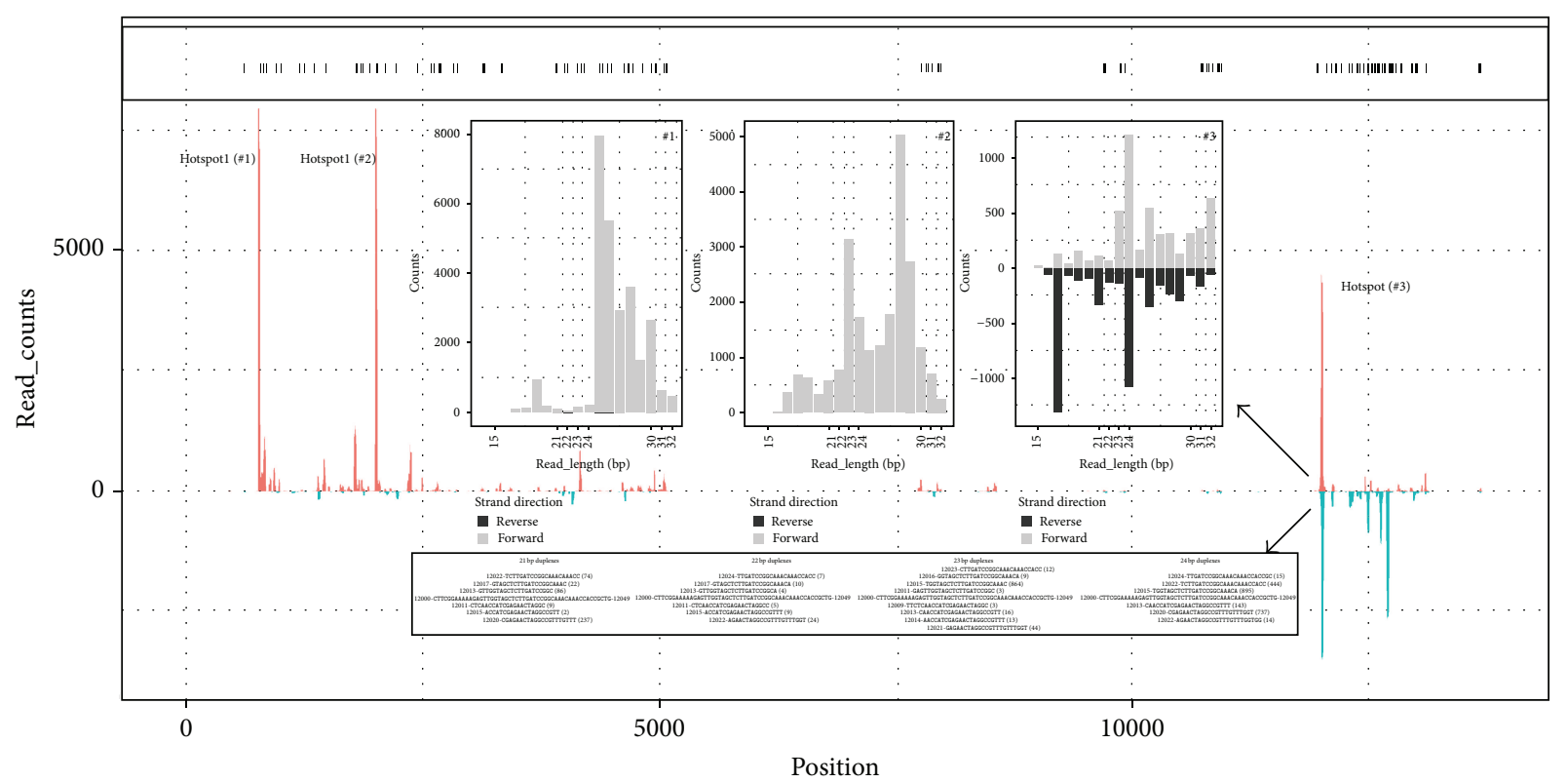

FIGURE 1: Nucleotide polymorphism, hotspots, and siRNA duplexes of HIV-1. The $x$-axis represents positions on the HIV-1 reference genome (GenBank: M19921). The $y$-axis represents the read counts from the data SRR941591 on each position. The dots in the top black box represent positions with polymorphic nucleotides. $\# 1, \# 2$, and \#3 are the size distributions of positive- and negative-strand viral reads in hotspot 1 (779$810 \mathrm{bp}$ ), hotspot 2 (2,017-2,045 bp), and hotspot 3 (12,006-12,044 bp). The read counts of $21 \mathrm{bp}, 22 \mathrm{bp}, 23 \mathrm{bp}$, and $24 \mathrm{bp}$ siRNA duplexes are marked in parentheses.

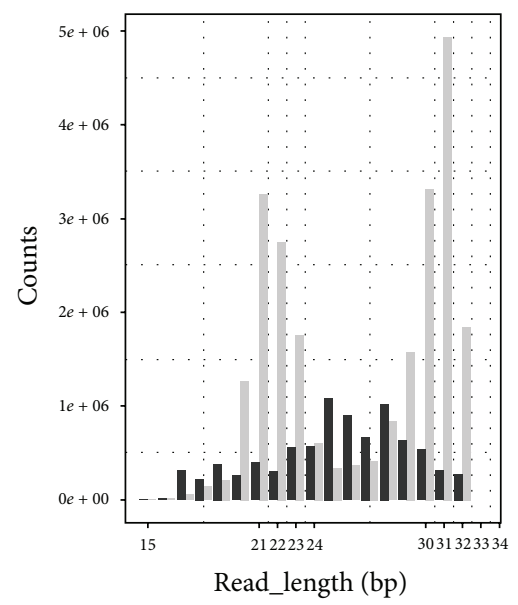

Read counts
HIV-1 $\times 100$ reads
All reads

(a)

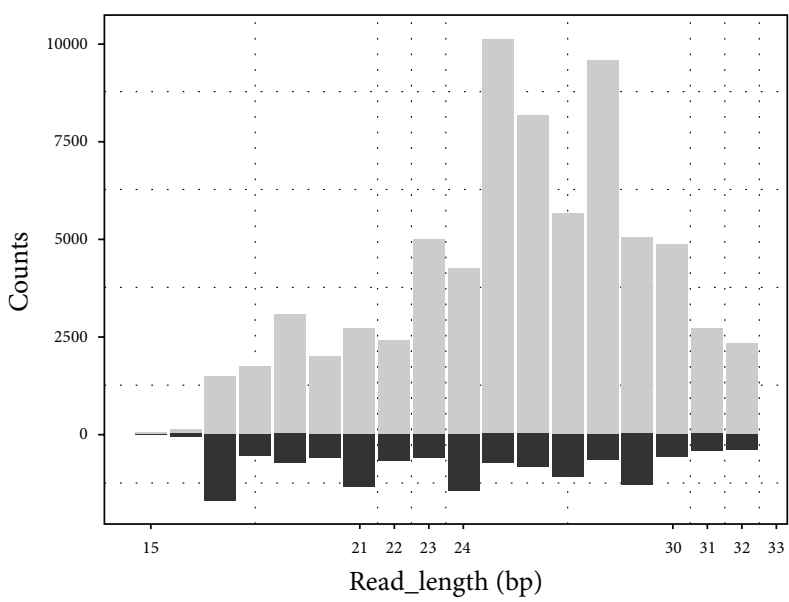

Strand direction

Reverse

Forward

(b)

FIGURE 2: Distribution of the total and HIV-1 viral read length on both of the strands. The $x$-axis represents read length. The $y$-axis represents the read counts of each length in the data SRR941591. HIV-1 $\times 100$ reads represent 100 times of reads which can be aligned to the HIV-1 reference genome (GenBank: M19921).

is that there must be positive- and negative-strand vsRNAs in some hotspots. In the data SRR941591, the third hotspot satisfied this criterion. The last and key step to identify RNAi responses is to find the siRNA duplexes from hotspots. They are usually only a minute fraction of the total vsRNAs, because the duplexes are short lived, due to one of the two strands being rapidly degraded following their creation. In the third hotspot, we found three canonical 22 bp siRNA duplexes containing a $20 \mathrm{nt}$ perfectly base-paired duplex region with $2 \mathrm{nt} 3^{\prime}$ overhangs. We also found 21, 23, and 24 bp siRNA-like duplexes, respectively (Figure 1). However, we used the putative HIV-1 reference M19921 for this analysis, 


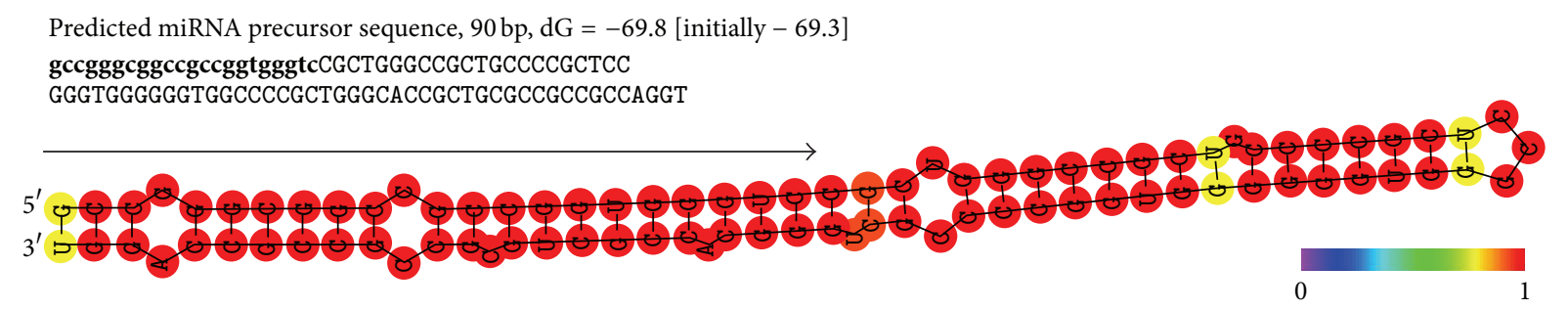

(a)

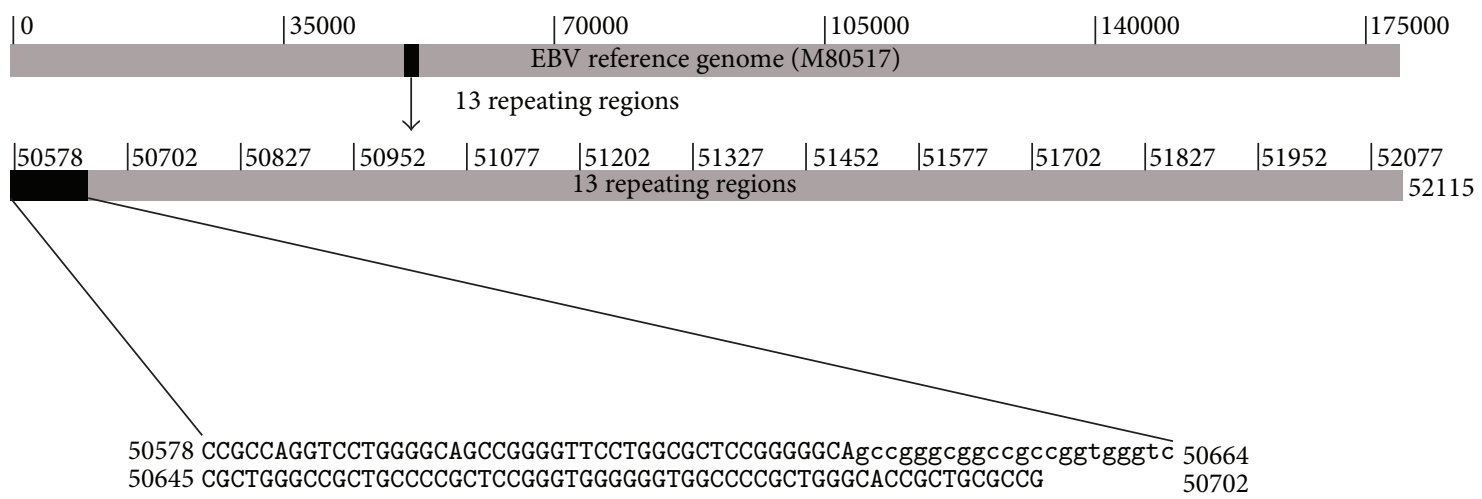

(b)

FIGURE 3: The predicted miRNAs of EBV. The EBV detected in data SRR1563063 is represented using the reference genome (GenBank: M80517) in this study. The sequence of the predicted mature miRNA is represented using the lowercase letters. (a) The second structures of the miRNA were predicted using RNAfold. (b) The first repeating unit (50578-50702) contains the predicted mature miRNA (50624-50646). This mature miRNA is repeated 12 times in 13 repeated units.

without knowledge of the exact HIV-1 sequence. M19921 is recombinant clone pNL4-3, which includes the HIV-1 virus region and vector region. Since the pNL4-3 clone is only constructed for the experiment use, the vsRNAs on the pNL4-3 vector region could be contamination during the library construction or sequencing process. Although the third hotspot existed in the pNL4-3 vector region, rather than the HIV-1 region on the reference M19921, the reliability of these siRNA duplexes in the third hotspot was supported by their uniqueness in the NCBI GenBank database and high sequencing depth. Therefore, this RNAi response could have happened in other samples.

In addition to the siRNAs, vsRNAs include other small RNA reads, for example, microRNAs (miRNAs), piwiinteracting RNAs (piRNAs), or degraded mRNA fragments. DNA viruses produce their own miRNAs facilitating the detection of DNA viruses, for example, EBV. In the data SRR1563063, we found seven miRNA-like duplexes from EBV vsRNAs (Supplementary File 3). Then, we blasted these duplexes to the miRBase database (http://www.mirbase.org/) and identified three known mature virus miRNAs. They were rlcv-mir-rL1-1-3p, ebv-mir-BART1-5p, and ebv-mir-BART13 p located at positions $53,801,151,640$, and 151,676 on the EBV reference M80517. Three mature miRNAs came from two miRNA precursors (pre-miRNAs) rlcv-mir-rL1-1 and ebvmir-BART1. Compared to two other miRNAs, the ebv-mirBART1-5p was expressed at a very high level of 17,987 read counts. As for the remaining four duplexes, we confirmed they could not be matched to the human genome. Then, we used RNAfold online server (http://rna.tbi.univie.ac.at/cgibin/RNAfold.cgi) to predict the second structures of their pre-miRNAs (Supplementary File 4). As a result, we identified a new EBV pre-miRNA, with a length of $90 \mathrm{bp}$ and a very high minimal folding free energy index (MEFI) over 1.0 (Figure 3(a)). Furthermore, this pre-miRNA resided in a repeating region on the reference M80517. This repeating region from 50,578 to 52,077 bp had 13 units (Figure 3(b)). Each unit with a length of $125 \mathrm{bp}$ contained this pre-miRNA sequence. It suggested that this repeating region comprise a primary miRNA (pri-miRNA).

\section{Conclusions}

In this study, we used 931 sRNA-seq runs of data from the NCBI SRA database to detect and identify human viruses. Six viruses were detected and two of them were not found in previous studies. These results suggest the sRNA-seq can be used to detect viruses in mammals and humans. The sRNA-seq data contains the heterozygosity information that can be used to investigate the pathogen evolution in one person and design therapies to deal with a specific virus population. The sRNA-seq data can also be used to find new virus miRNA or to investigate the RNAi responses in mammals and humans. However, sRNA-seq data used in this study were not from virus-infection experiments with knowledge of the exact virus sequences. In place of the exact virus sequences, the putative virus sequences were used to investigate the RNAi responses. Although using the putative 
virus sequences brought some uncertainties, the results of this study still shed light on the studies of virus induced RNAi in mammals.

\section{Conflict of Interests}

The authors declare that no financial competing interests exist.

\section{Authors' Contribution}

Shan Gao conceived the project. Shan Gao and Xiao Zhu supervised this study. Shan Gao wrote the main paper text. Jan F. Kreuze and Jishou Ruan revised the paper. Fang Wang, Yu Sun, and Rui Chen downloaded, managed, and processed the data. Xin Chen prepared the figures and tables. Chengjie Chen conducted programming. Zhangjun Fei gave suggestion to build the virus detection pipeline. Fang Wang and Yu Sun contributed equally to this paper.

\section{Acknowledgments}

The authors appreciate the help equally from the people listed below. They are Associate Professor Jijun Tang from the Department of Computer Science \& Engineering, University of South Carolina, Associate Professor Xiujun Gong from the College of Computer Science \& Tech, Tianjin University, and Professor Wenjun $\mathrm{Bu}$ from the College of Life Sciences, Nankai University. The data analysis in this study was supported by the National Scientific Data Sharing Platform for Population and Health Translational Cancer Medicine Specials. This work was supported partly by the National Natural Science Foundation of China (81541153), Guangdong Provincial Research Project of Science and Technology (2015A050502048 and 2014A020212295), and Science and Technology Research Project in Dongguan City (2013508152011 and 2013508152002).

\section{References}

[1] A. A. Ansari, "Clinical features and pathobiology of Ebolavirus infection," Journal of Autoimmunity, vol. 55, pp. 1-9, 2014.

[2] K. Neyt and B. N. Lambrecht, "The role of lung dendritic cell subsets in immunity to respiratory viruses," Immunological Reviews, vol. 255, no. 1, pp. 57-67, 2013.

[3] G. F. Wang, W. Li, and K. Li, "Acute encephalopathy and encephalitis caused by influenza virus infection," Current Opinion in Neurology, vol. 23, no. 3, pp. 305-311, 2010.

[4] A. A. Lackner, M. Mohan, and R. S. Veazey, "The gastrointestinal tract and aids pathogenesis," Gastroenterology, vol. 136, no. 6, pp. 1966-1978, 2009.

[5] C. De Martel, J. Ferlay, S. Franceschi et al., "Global burden of cancers attributable to infections in 2008: a review and synthetic analysis," The Lancet Oncology, vol. 13, no. 6, pp. 607-615, 2012.

[6] O. Isakov, S. Modai, and N. Shomron, "Pathogen detection using short-RNA deep sequencing subtraction and assembly," Bioinformatics, vol. 27, no. 15, pp. 2027-2030, 2011.

[7] G. M. Daly, N. Bexfield, J. Heaney et al., "A viral discovery methodology for clinical biopsy samples utilising massively parallel next generation sequencing," PLoS ONE, vol. 6, no. 12, Article ID e28879, 2011.

[8] J. F. Kreuze, A. Perez, M. Untiveros et al., "Complete viral genome sequence and discovery of novel viruses by deep sequencing of small RNAs: a generic method for diagnosis, discovery and sequencing of viruses," Virology, vol. 388 , no. 1 , pp. 1-7, 2009.

[9] S. Mlotshwa, G. J. Pruss, and V. Vance, "Small RNAs in viral infection and host defense," Trends in Plant Science, vol. 13, no. 7, pp. 375-382, 2008.

[10] B. R. Cullen, S. Cherry, and B. R. Tenoever, "Is RNA interference a physiologically relevant innate antiviral immune response in mammals?" Cell Host \& Microbe, vol. 14, no. 4, pp. 374-378, 2013.

[11] C. Hagen, A. Frizzi, J. Kao et al., "Using small RNA sequences to diagnose, sequence, and investigate the infectivity characteristics of vegetable-infecting viruses," Archives of Virology, vol. 156, no. 7, pp. 1209-1216, 2011.

[12] R. G. Li, S. Gao, A. G. Hernandez, W. P. Wechter, Z. J. Fei, and K.-S. Ling, "Deep sequencing of small RNAs in tomato for virus and viroid identification and strain differentiation," PLOS ONE, vol. 7, no. 5, Article ID e37127, 2012.

[13] A. Nayak, M. Tassetto, M. Kunitomi, and R. Andino, "RNA interference-mediated intrinsic antiviral immunity in invertebrates," in Intrinsic Immunity, pp. 183-200, Springer, 2013.

[14] Q. Wu, Y. Luo, R. Lu et al., "Virus discovery by deep sequencing and assembly of virus-derived small silencing RNAs," Proceedings of the National Academy of Sciences of the United States of America, vol. 107, no. 4, pp. 1606-1611, 2010.

[15] V. Gausson and M.-C. Saleh, "Viral small RNA cloning and sequencing," Methods in Molecular Biology, vol. 721, pp. 107-122, 2011.

[16] Y. Li, J. Lu, Y. Han, X. Fan, and S.-W. Ding, "RNA interference functions as an antiviral immunity mechanism in mammals," Science, vol. 342, no. 6155, pp. 231-234, 2013.

[17] R. Leinonen, H. Sugawara, and M. Shumway, "The sequence read archive," Nucleic Acids Research, vol. 39, no. 1, pp. D19-D21, 2011.

[18] M. Zhang, H. Sun, Z. Fei, F. Zhan, X. Gong, and S. Gao, "Fastqclean: an optimized pipeline to clean the illumina sequencing data with quality control," in Proceedings of the IEEE International Conference on Bioinformatics and Biomedicine (BIBM '14), pp. 44-48, IEEE, Belfast, Northern Ireland, November 2014.

[19] R. G. Li, S. Gao, Z. J. Fei, and K. S. Ling, "Complete genome sequence of a new tobamovirus naturally infecting tomatoes in Mexico," Genome Announcements, vol. 1, no. 5, Article ID e00794-13, 2013.

[20] C. Padmanabhan, S. Gao, R. Li, S. Zhang, Z. Fei, and K.-S. Ling, "Complete genome sequence of an emerging genotype of tobacco streak virus in the united states," Genome Announcements, vol. 2, no. 6, Article ID e01138-14, 2014.

[21] R. G. Li, S. Gao, S. Berendsen, Z. J. Fei, and K. S. Ling, "Complete genome sequence of a novel genotype of squash mosaic virus infecting Squash in Spain," Genome Announcements, vol. 3, no. 1, Article ID e01583-14, 2015.

[22] C. Quast, E. Pruesse, P. Yilmaz et al., "The SILVA ribosomal RNA gene database project: Improved data processing and webbased tools," Nucleic Acids Research, vol. 41, no. 1, pp. D590D596, 2013.

[23] P. Arbuthnot and M. Kew, "Hepatitis B virus and hepatocellular carcinoma," International Journal of Experimental Pathology, vol. 82, no. 2, pp. 77-100, 2001. 
[24] J. Hou, L. Lin, W. Zhou et al., "Identification of miRNomes in human liver and hepatocellular carcinoma reveals miR-199a/b$3 \mathrm{p}$ as therapeutic target for hepatocellular carcinoma," Cancer Cell, vol. 19, no. 2, pp. 232-243, 2011.

[25] S. Buttò, B. Suligoi, E. Fanales-Belasio, and M. Raimondo, "Laboratory diagnostics for HIV infection," Annali dell'Istituto Superiore di Sanità, vol. 46, no. 1, pp. 24-33, 2010.

[26] X. Wu, G. Somlo, Y. Yu et al., "De novo sequencing of circulating miRNAs identifies novel markers predicting clinical outcome of locally advanced breast cancer," Journal of Translational Medicine, vol. 10, no. 1, article 42, 2012.

[27] D. Colcher, R. L. Heberling, S. S. Kalter, and J. Schlom, "Squirrel monkey retrovirus: an endogenous virus of a new world primate," Journal of Virology, vol. 23, no. 2, pp. 294-301, 1977.

[28] C. C. Uphoff, S. A. Denkmann, K. G. Steube, and H. G. Drexler, "Detection of EBV, HBV, HCV, HIV-1, HTLV-I andII, and SMRV in human and other primate cell lines," Journal of Biomedicine and Biotechnology, vol. 2010, Article ID 904767, 2010.

[29] P. G. Middleton, S. Miller, J. A. Ross, C. M. Steel, and K. Guy, "Insertion of SMRV-H viral DNA at the c-myc gene locus of a BL cell line and presence in established cell lines," International Journal of Cancer, vol. 52, no. 3, pp. 451-454, 1992.

[30] C. Y. Ok, T. G. Papathomas, L. J. Medeiros, and K. H. Young, "EBV-positive diffuse large B-cell lymphoma of the elderly," Blood, vol. 122, no. 3, pp. 328-340, 2013.

[31] L. Arcaini, D. Rossi, M. Lucioni et al., "The NOTCH pathway is recurrently mutated in diffuse large B-cell lymphoma associated with hepatitis C virus infection," Haematologica, vol. 100, no. 2, pp. 246-252, 2015.

[32] K. Liapis, A. Clear, A. Owen et al., "The microenvironment of AIDS-related diffuse large B-cell lymphoma provides insight into the pathophysiology and indicates possible therapeutic strategies," Blood, vol. 122, no. 3, pp. 424-433, 2013.

[33] S.-I. Nakatsuka, A. Liu, Z. Dong et al., "Simian virus 40 sequences in malignant lymphomas in Japan," Cancer Research, vol. 63, no. 22, pp. 7606-7608, 2003.

[34] D. A. Thorley-Lawson and M. J. Allday, "The curious case of the tumour virus: 50 years of Burkitt's lymphoma," Nature Reviews Microbiology, vol. 6, no. 12, pp. 913-924, 2008.

[35] D. Kutnjak, M. Rupar, I. Gutierrez-Aguirre, T. Curk, J. F. Kreuze, and M. Ravnikar, "Deep sequencing of virus-derived small interfering RNAs and RNA from viral particles shows highly similar mutational landscapes of a plant virus population," Journal of Virology, vol. 89, no. 9, pp. 4760-4769, 2015.

[36] J. D. Roberts, K. Bebenek, and T. A. Kunkel, "The accuracy of reverse transcriptase from HIV-1," Science, vol. 242, no. 4882, pp. 1171-1173, 1988. 

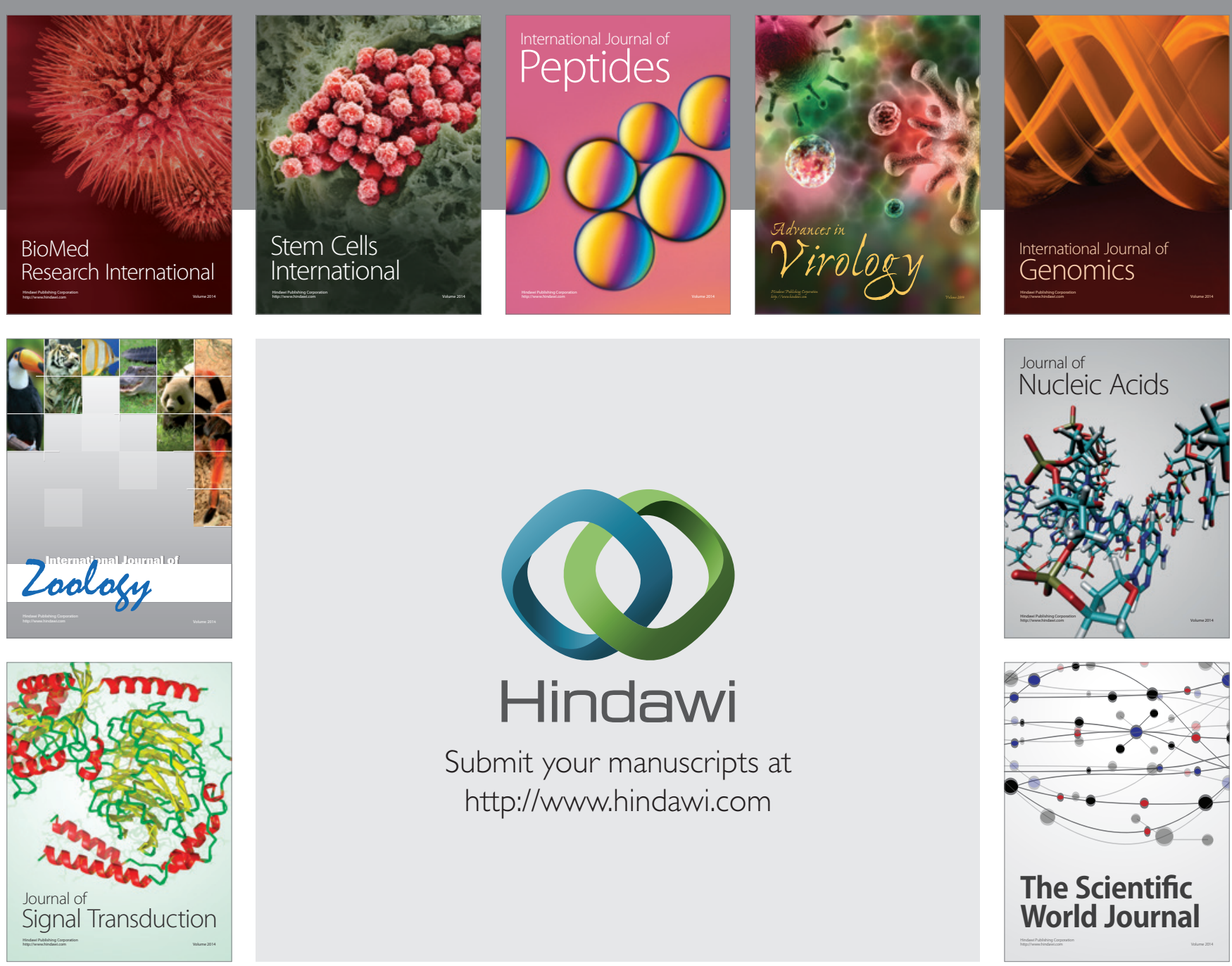

Submit your manuscripts at

http://www.hindawi.com
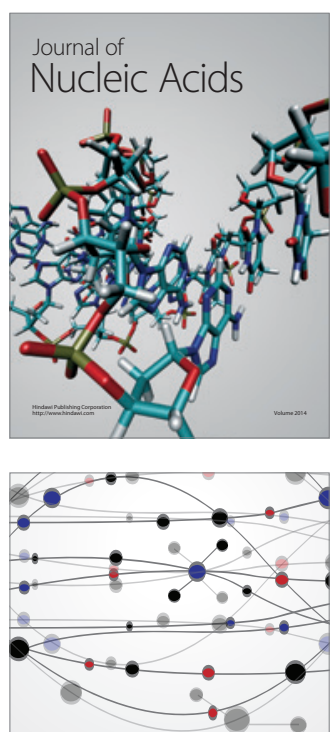

The Scientific World Journal
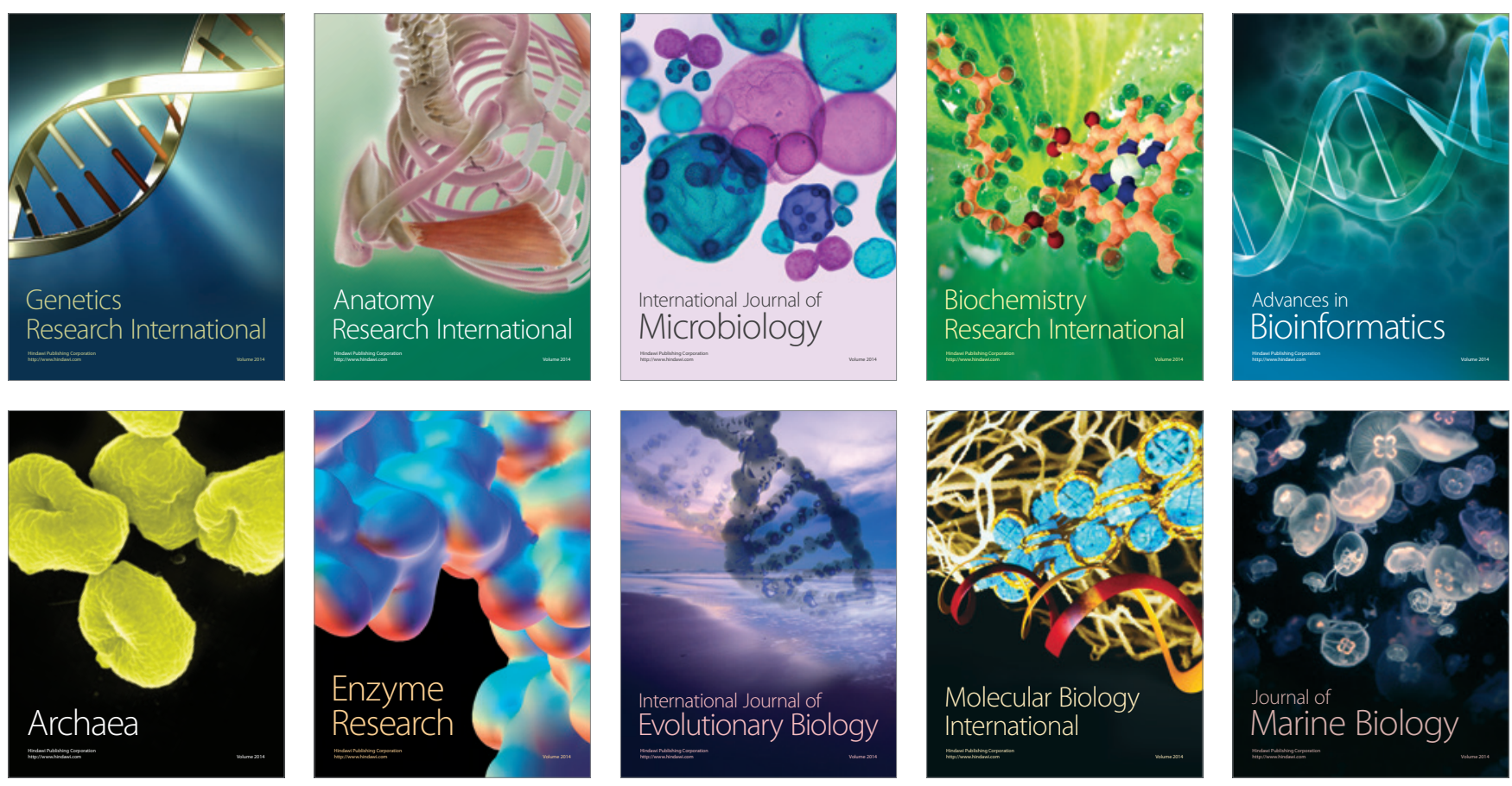